\title{
Representation of a Gradient for Scalar Electromagnetic Field in MATLAB using Differential Operators
}

\author{
D.K. Bonello ${ }^{1 *}$, Y. Iano ${ }^{2}$, U.B. Neto ${ }^{3}$ \\ ${ }^{1}$ Dept. of Communications, School of Electrical and Computer Engineering, University of Campinas, Campinas, Brazil \\ ${ }^{2}$ Dept. of Communications, School of Electrical and Computer Engineering, University of Campinas, Campinas, Brazil \\ ${ }^{3}$ Dept. of Communications, School of Electrical and Computer Engineering, University of Campinas, Campinas, Brazil
}

*Corresponding Author: danielb.katze@gmail.com, Tel.: +55-19-999699274

Available online at: www.isroset.org

Received: 30/Mar/2020, Accepted: 13/Apr/2020, Online: 30/Apr/2020

\begin{abstract}
In this work was deduced the representation of an algorithm, developed in MATLAB, to calculate an electromagnetic scalar field utilizing differential operators to generate graphics about the electromagnetic spectrum, the distance $\mathrm{X}, \mathrm{Y}$ of the field curvature, as well as the gradient example of the electromagnetic field. The main objective of this article is to compare the new GUI features of the algorithm developed in MATLAB to the previous algorithm as cited in the previous work of this paper, showing also the spectrum average of this new algorithm can reach, according to the differential operators values inserted into the code.
\end{abstract}

Keywords_Electromagnetic field, Differential operators, Numerical calculus, Simulations, MATLAB, Linear function

\section{INTRODUCTION}

Electromagnetic fields were first discovered in the 19th century, when physicists noticed that electric arcs (sparks) could be reproduced at a distance, with no connecting wires in between. This led scientists to believe that it was possible to communicate over long distances without wires [1].

An electromagnetic field, sometimes referred to as an EM field, is generated when charged particles, such as electrons, are accelerated, being all electrically charged particles are surrounded by electric fields. Charged particles in motion produce magnetic fields. When the velocity of a charged particle changes, an EM field is produced.

With a title of example, regarding the gradient of scalar fields, let us consider a metal bar whose temperature varies from point to point in some complicated manner. So, the temperature will be a function of $\mathrm{x}, \mathrm{y}, \mathrm{z}$ in the Cartesian coordinate system. Hence temperature here is a scalar field represented by the function $\mathrm{T}(\mathrm{x}, \mathrm{y}, \mathrm{z})$. Since temperature depends on the distance it could increase in some directions and decrease in some directions. It could increase or decrease rapidly along with some directions in comparison to other directions [2].

Hence, the gradient of a scalar field is a vector field and whose magnitude is the rate of change and which points in the direction of the greatest rate of increase of the scalar field. If the vector is resolved, its components represent the rate of change of the scalar field with respect to each directional component [3].
This paper is structured presenting a related work showing the equations of scalar fields utilized in the MATLAB simulations, the methodology utilized to obtain the new based GUI algorithm, the results and discussion appointed the main contributions of this work and finally the conclusions.

\section{RELATED WORK}

For a two-dimensional scalar field $\varnothing(\mathrm{x}, \mathrm{y})$, we have the Equation (1) [4]:

$$
\operatorname{grad} \phi(x, y)=\nabla \phi(x, y)=\left(\frac{\partial}{\partial x}, \frac{\partial}{\partial y}\right) \phi=\left(\frac{\partial \phi}{\partial x}, \frac{\partial \phi}{\partial y}\right)
$$

And for a three-dimensional scalar field $\varnothing(\mathrm{x}, \mathrm{y}, \mathrm{z})$ we have the Equation (2):

$$
\operatorname{grad} \phi(x, y, z)=\nabla \phi(x, y, z)=\left(\frac{\partial}{\partial x}, \frac{\partial}{\partial y}, \frac{\partial}{\partial z}\right) \phi=\left(\frac{\partial \phi}{\partial x}, \frac{\partial \phi}{\partial y}, \frac{\partial \phi}{\partial z}\right)
$$

The gradient of a scalar field is the derivative of $\mathrm{f}$ in each direction. Note that the gradient of a scalar field is a vector field. An alternative notation is to use the $d e l$ or nabla operator, $\nabla \mathrm{f}=\operatorname{grad} \mathrm{f}$.

For a three dimensional scalar, its gradient is given by the Equation (3):

$$
\operatorname{grad}(V)=\underline{a}_{n} \frac{d V}{d n}=\nabla V
$$


The gradient is a vector that represents both the magnitude and the direction of the maximum space rate of increase of a scalar.

$$
d V=(\nabla V) \cdot d \underline{l}, \text { where } d \underline{l}=\underline{a}_{i} \cdot d \underline{l}
$$

In a Cartesian plane, we represent the gradient function according to the Equation (5):

$$
\nabla \equiv \underline{a}_{x} \frac{\partial}{\partial x}+\underline{a}_{y} \frac{\partial}{\partial y}+\underline{a}_{z} \frac{\partial}{\partial z}
$$

In a Cylindrical plane, we represent the gradient function according to the Equation (6):

$$
\nabla \equiv \underline{a}_{r} \frac{\partial}{\partial r}+\underline{a}_{\phi} \frac{\partial}{r \cdot \partial \phi}+\underline{a}_{z} \frac{\partial}{\partial z}
$$

In a Spherical plane, we represent the gradient function according to the Equation (7):

$$
\nabla \equiv \underline{a}_{R} \frac{\partial}{\partial R}+\underline{a}_{\theta} \frac{\partial}{R \partial \theta}+\underline{a}_{\theta} \frac{\partial}{R \cdot \sin \theta \cdot \partial \phi}
$$

\section{METHODOLOGY}

The following image shows the screen of the algorithm developed in MATLAB capable to detect a gradient of a scalar electromagnetic field using differential operators $\nabla$ $(\mathrm{x}, \mathrm{y}, \mathrm{z})$ within four minimal differential functions defined as the patterns for this study in question.

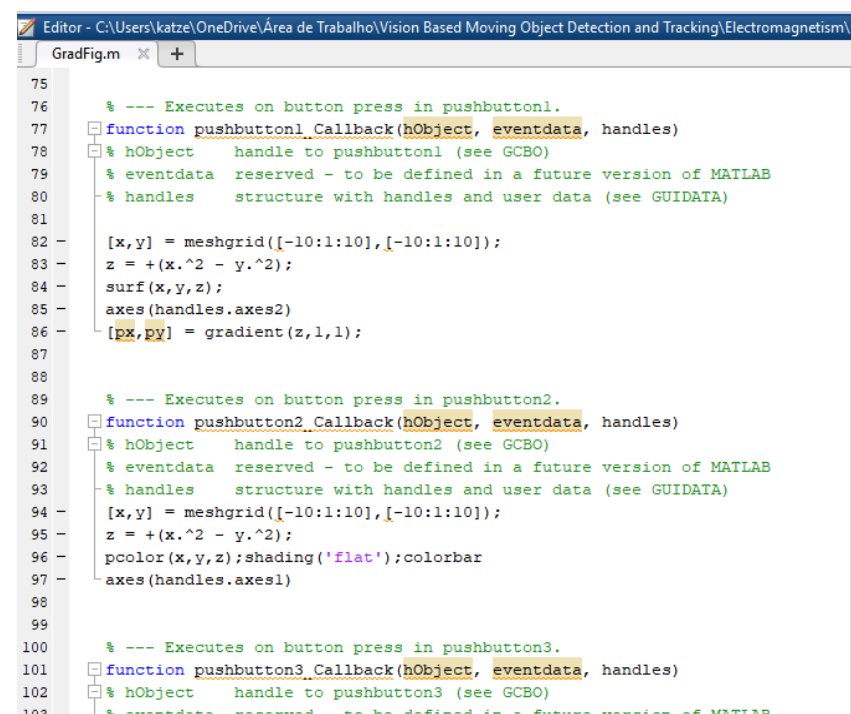

Figure 1. Algorithm for scalar electromagnetic field representation developed in MATLAB.

Also can be written in Table 1 the differential operators $\mathrm{z}$ $=(\mathrm{xn}+\mathrm{ym})$ for detection the gradient of the scalar electromagnetic field that will be represented by the graphics of the electromagnetic spectrum, the distance $\mathrm{X}, \mathrm{Y}$ of the field curvature, as well as the gradient example inserts in the GUI developed in MATLAB.

Table 1. Differential operators considered for simulation
\begin{tabular}{|c|c|}
\hline Equation number & Differential operator \\
\hline 1 & $\mathrm{z}=-\left(\mathrm{x}^{2}+\mathrm{y}^{2}\right)$ \\
\hline 2 & $\mathrm{z}=+\left(\mathrm{x}^{2}+\mathrm{y}^{2}\right)$ \\
\hline 3 & $\mathrm{z}=-\left(\mathrm{x}^{2}-\mathrm{y}^{2}\right)$ \\
\hline 4 & $\mathrm{z}=+\left(\mathrm{x}^{2}-\mathrm{y}^{2}\right)$ \\
\hline
\end{tabular}

We can see the new algorithm proposed for representation of the gradient of a scalar the electromagnetic field in MATLAB has an optimized interface with "Calculate $\mathrm{X}$ and Y distance", "Calculate vectors" and "Calculate spectrum" buttons inserted to the code as shows Figure 2:

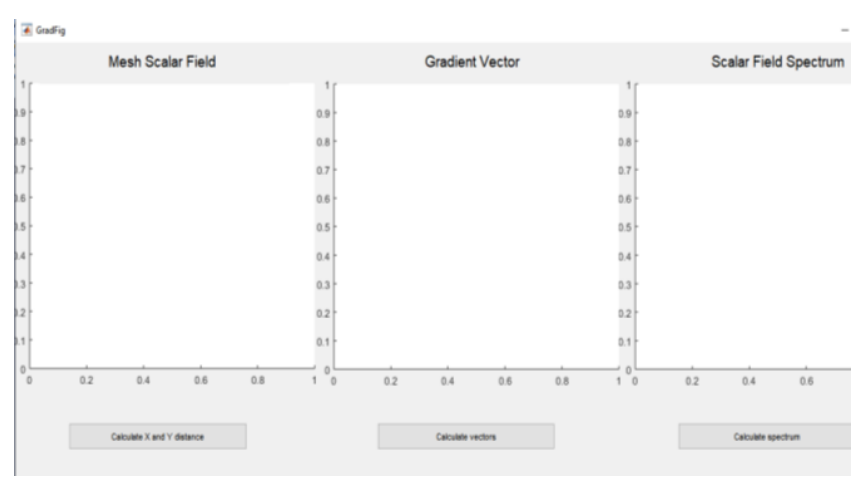

Figure 2. Improved GUI interface of he algorithm

The previous interfaces, as well as the previous algorithm for the electromagnetic field detection, is represented by the Figure 3, Figure 4 and Figure 5, as a simple "figure function" embedded at the previous code developed in MATLAB.

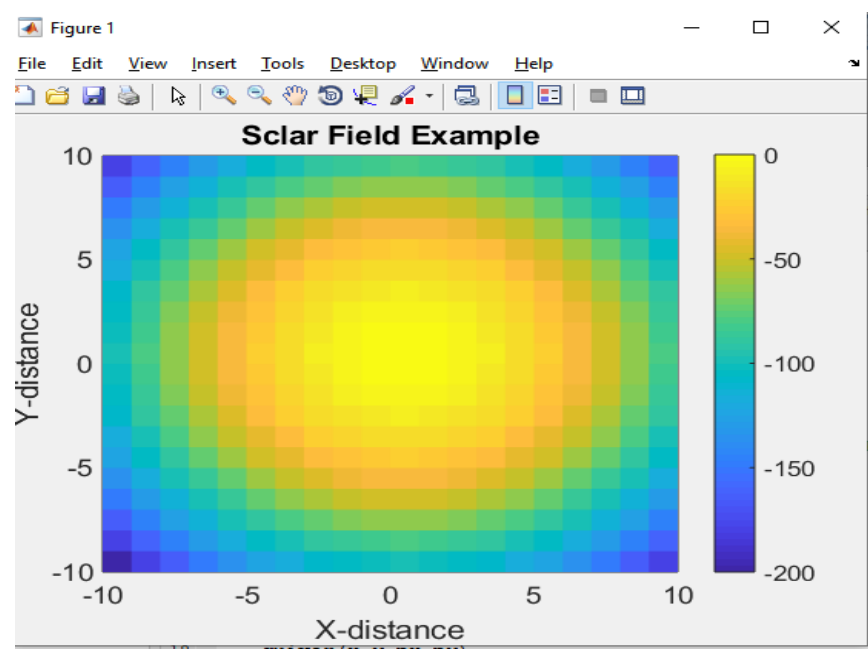

Figure 3. Scalar field example of the previous algorithm ussing "Figure interface" in MATLAB 


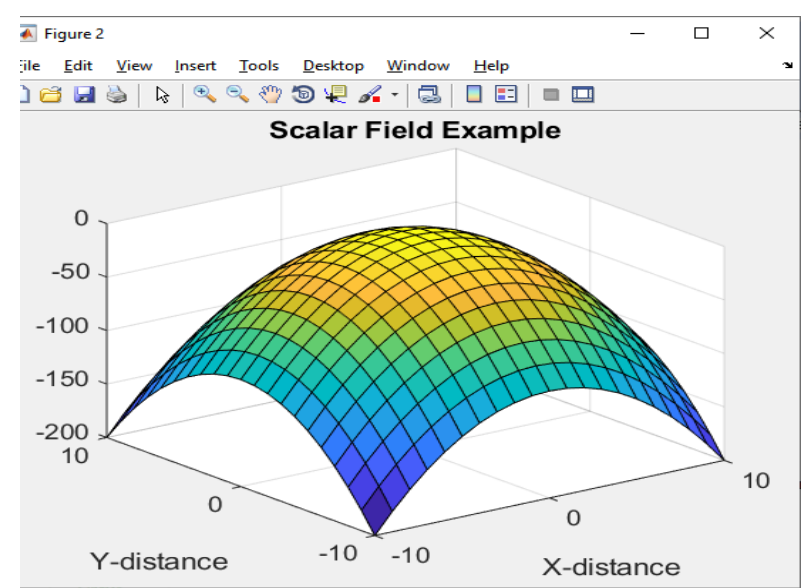

Figure 4. Curvature of the Scalar field example of the previous algorithm using "Figure interface" in MATLAB

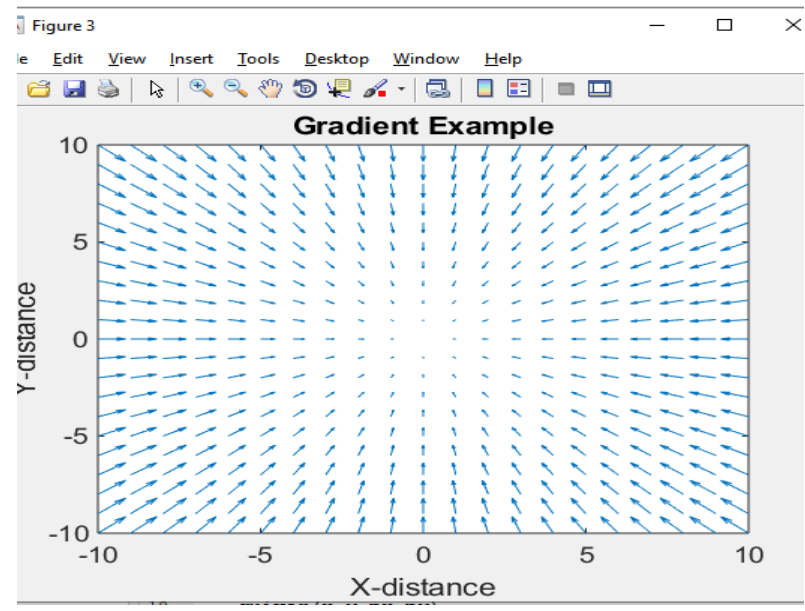

Figure 5. Gradient example of the previous algorithm using "Figure interface" in MATLAB

The previous algorithm also can be written below:

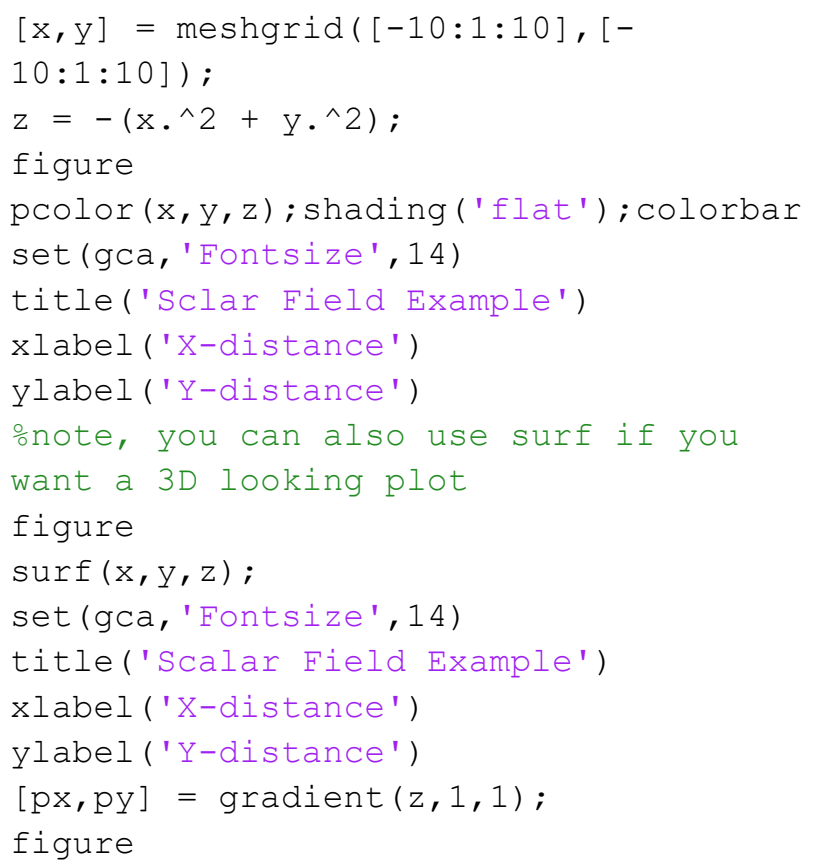

quiver $(\mathrm{x}, \mathrm{y}, \mathrm{px}, \mathrm{py})$

set (gca, 'Fontsize', 14)

title('Gradient Example')

xlabel ('X-distance')

ylabel ('Y-distance')

The new GUI interface developed in MATLAB for the representation of the gradient for a scalar electromagnetic field, using the differential operators showed in Table 1, are shown below for each case in the simulations:

- $z=-\left(x^{2}+y^{2}\right)$

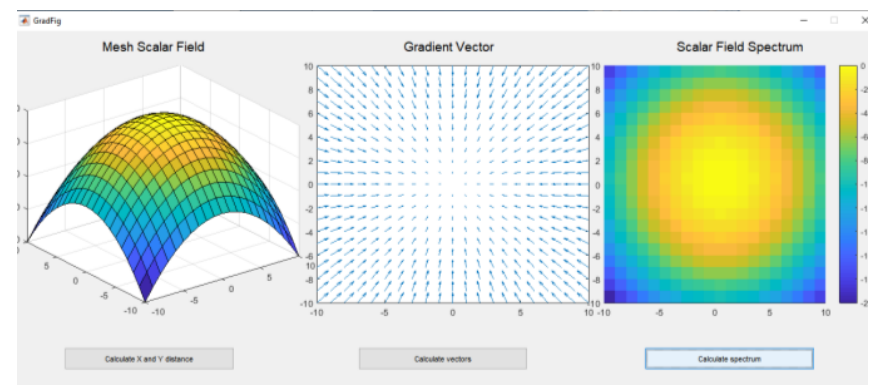

Figure 6. Electromagnetic field represented by the differential operator $\mathrm{z}=-\left(\mathrm{x}^{2}+\mathrm{y}^{2}\right)$

- $\mathrm{z}=+\left(\mathrm{x}^{2}+\mathrm{y}^{2}\right)$

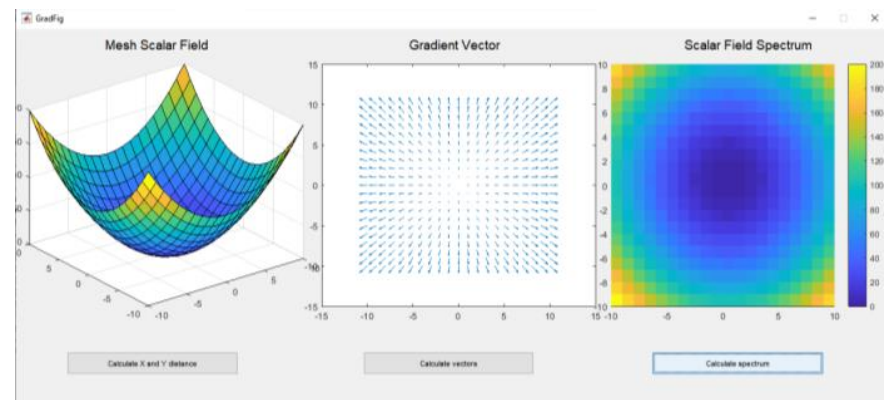

Figure 7. Electromagnetic field represented by the differential operator $\mathrm{z}=+\left(\mathrm{x}^{2}+\mathrm{y}^{2}\right)$

- $z=-\left(x^{2}-y^{2}\right)$

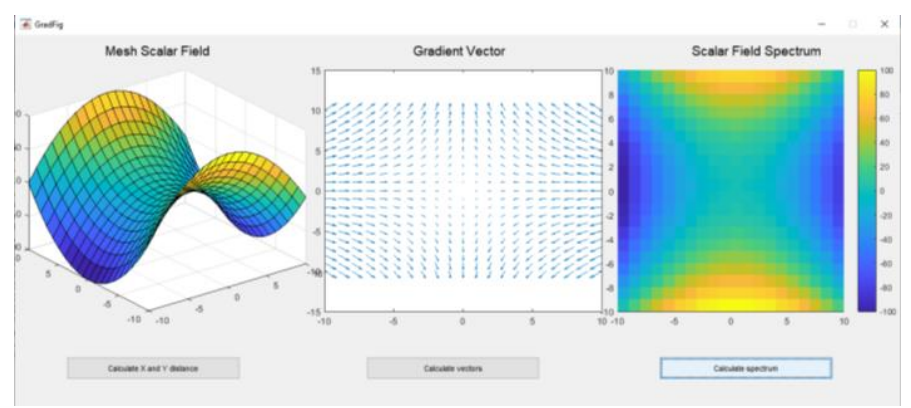

Figure 8. Electromagnetic field represented by the differential operator $\mathrm{z}=-\left(\mathrm{x}^{2}-\mathrm{y}^{2}\right)$

- $\quad \mathrm{z}=+\left(\mathrm{x}^{2}-\mathrm{y}^{2}\right)$ 


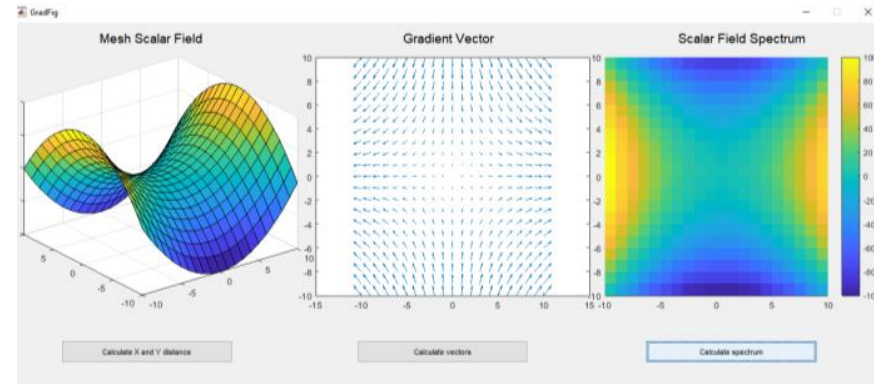

Figure 9. Electromagnetic field represented by the differential operator $\mathrm{z}=+\left(\mathrm{x}^{2}-\mathrm{y}^{2}\right)$

\section{RESULTS AND DISCUSSION}

The results appoint a maximum electromagnetic field value about $200 \mathrm{~kg} . \mathrm{s}^{-2} . \mathrm{A}^{-1}$ and a minimum value about $200 \mathrm{~kg} \cdot \mathrm{s}^{-2} \cdot \mathrm{A}^{-1}$ when we insert the differential equations $\mathrm{z}=+\left(\mathrm{x}^{2}+\mathrm{y}^{2}\right)$ and $\mathrm{z}=-\left(\mathrm{x}^{2}+\mathrm{y}^{2}\right)$, respectively, to the new algorithm developed in MATLAB. Those values represent the spectrum of the electromagnetic field in the SI unit. This means a directly proportional greatness in relation to the distance $(\mathrm{m})$ and value $\left(\mathrm{kg} \cdot \mathrm{s}^{-2} \cdot \mathrm{A}^{-1}\right)$ of the electromagnetic field spectrum. Hence, for the differential operators $\mathrm{z}=+\left(\mathrm{x}^{2}+\mathrm{y}^{2}\right)$ and $\mathrm{z}=-\left(\mathrm{x}^{2}+\mathrm{y}^{2}\right)$, as larger as the distance from the point "zero", less intense is the electromagnetic field. The Graphic 1 and Graphic 2, indicated in the Figure 10 and Figure 11, respectively, shows the linear functions of the differential operators $\mathrm{z}=$ $+\left(x^{2}+y^{2}\right)$ and $z=-\left(x^{2}+y^{2}\right)$.

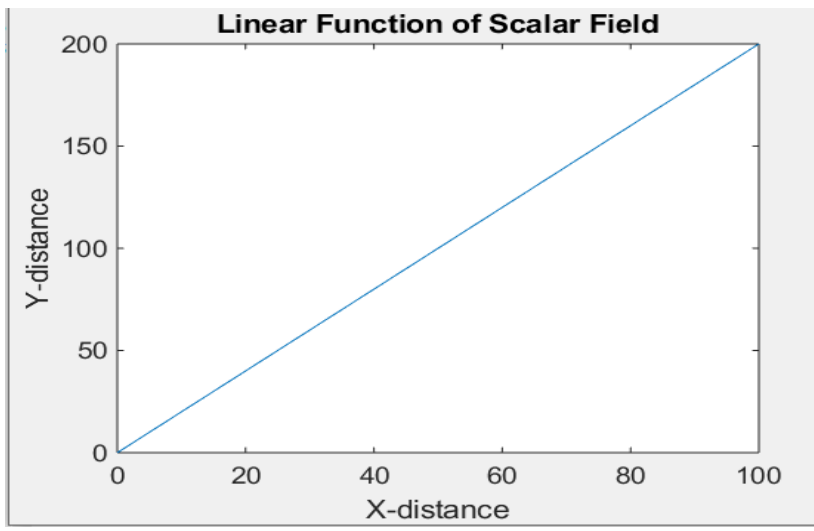

Figure 10. Electromagnetic linear function represented by the differential operator $\mathrm{z}=+\left(\mathrm{x}^{2}+\mathrm{y}^{2}\right)$

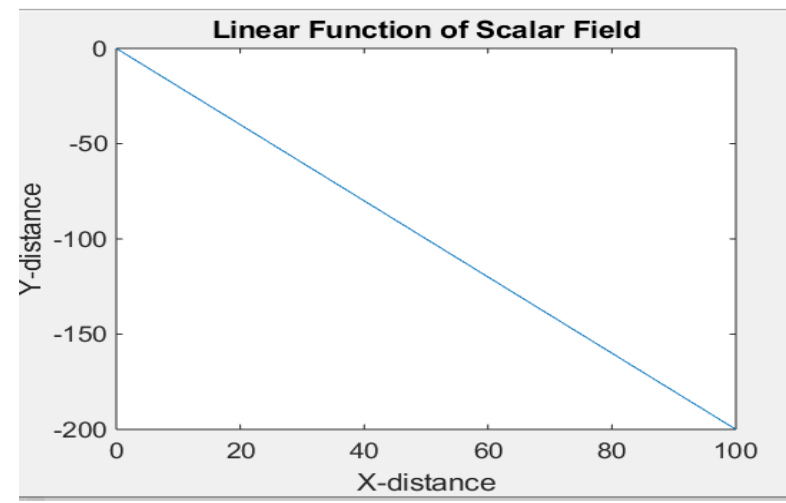

Figure 11. Electromagnetic linear function represented by the differential operator $\mathrm{z}=-\left(\mathrm{x}^{2}+\mathrm{y}^{2}\right)$
Graphic 3 and Graphic 4 also can be plotted to the differential operators $z=+(x 2-y 2)$ and $z=-(x 2-y 2)$, indicating proportional greatness in relation to the distance (m) and value (T) of the electromagnetic field spectrum.

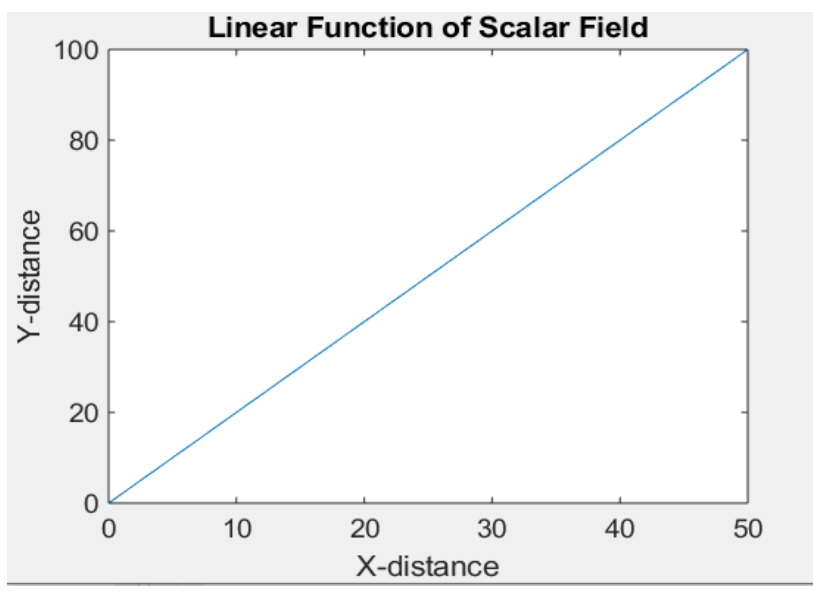

Figure 12. Positive electromagnetic linear function represented by the differential operator $\mathrm{z}=+\left(\mathrm{x}^{2}-\mathrm{y}^{2}\right)$ and $\mathrm{z}=-\left(\mathrm{x}^{2}-\mathrm{y}^{2}\right)$

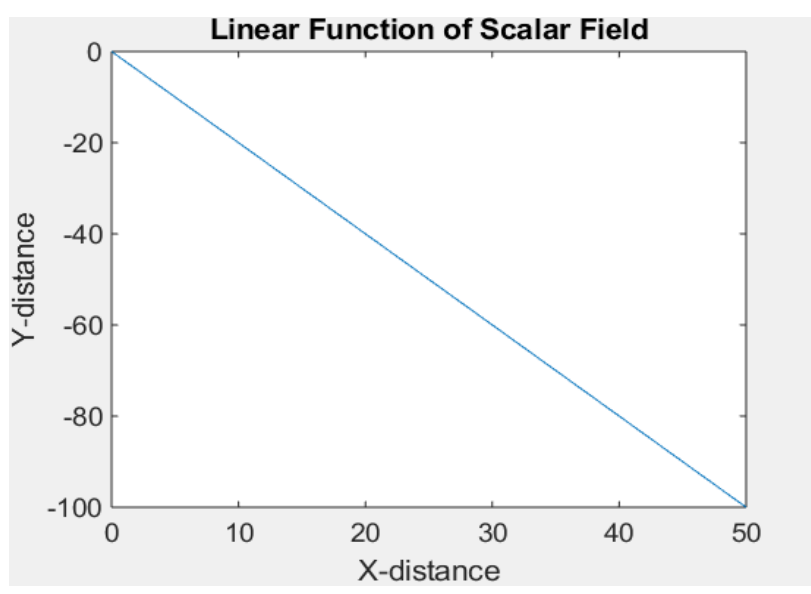

Figure 13. Negative electromagnetic linear function represented by the differential operator $\mathrm{z}=+\left(\mathrm{x}^{2}-\mathrm{y}^{2}\right)$ and $\mathrm{z}=-\left(\mathrm{x}^{2}-\mathrm{y}^{2}\right)$

\section{Discussion}

The idea of this article is to provide to the reader a general vision about the basics phenomena involved in the electromagnetic spectrum utilizing differential operators capable to represent gradient vectors and the X,Y curvature of the electromagnetic field in MATLAB. It is important the reader has a basic knowledge about the electromagnetism metrics, because those are the elements respond to the gradient vector generation. The presentation of the elements related to the electromagnetic field representation in this paper allows that your modeling can be incorporated into the other techniques of field representation, such as $3 \mathrm{D}$ images. The literature regarding the circle detection systems is very vast.

\section{CONCLUSION AND Future SCOPE}

In this work was deduced a model commonly utilized in the field of numerical calculus to represent an 
electromagnetic field (X,Y distance; spectrum and gradient vectors) of four main differential equations insert to the code developed in MATLAB. Once defined the new based GUI algorithm and the spectrum ratio detection in the code, were defined optimized implementation strategies for the linear functions for the scalar fields equations with a scale selection dependents of the spectrum average a representation showed in the new GUI Figures plotted in the sections above. After a definition of an optimization metric through the new based algorithm for electromagnetic field representation using differential operators, was verified the correlation between the distance from the electromagnetic spectrum $(\mathrm{m})$ and the intensity of the electromagnetic field $\left(\mathrm{kg} \cdot \mathrm{s}^{-2} \cdot \mathrm{A}^{-1}\right)$. With these results, was possible obtain also four linear graphics in the function of the differential operators utilized in this work to calculate the electromagnetic field spectrum.

\section{ACKNOWLEDGMENT}

The research was realized in the Laboratory of Communications at the University of Campinas.

\section{REFERENCES}

[1] M. Rouse, "Electromagnetic Field," TechTarget Publisher, United States, pp. 1-3, 2010.

[2] Portland State University, "ECE331 Lab 4: Numerical Electromagnetic Experiments," Department of Electrical and Computer Engineering, United States, pp. 2-8, 2013.

[3] Physics Catalyst, "Gradient of a scalar field and its physical significance," Physics Catalyst, United States, pp. 7-9, 2018.

[4] Web Formulas, "Gradient of a Scalar Field," Web Formulas, United States, pp.4-6, 2019.

[5] F. Anselmet, H. Djeridi, L. Furlachier, "Joint Statistics of a Passive Scalar and its Dissipation in Turbulent Flows," International Journal of Fluid Mech, Vol. 280, Issue.1, pp. 173197, 1994.

[6] E. Effelsberg, N. Peters, "A Composite Model for the Conserved Scalar PDF," International Journal of Combust Flame, Vol.50, Issue.1, pp. 351-360, 1983.

[7] J.H. Williamson, "Low-Storage Runge-Kutta Schemes," International Journal of Computer Physics, Vol.35, Issue.4, pp. $48-56,1980$.

[8] K.R. Sreenivasan, R.A. Antonia, "The Phenomenology of Small-Scale Turbulence," Апnи. Rev. Fluid Mech, United States, pp.435-472, 1997.

[9] C. Pantano, "Direct Simulation of Nonpremixed Flame Extinction in a Methane-Air Jet With Reduced Chemistry," International Journal of Fluid Mech, Vol.514, Issue.2, pp. 231270, 2004.

[10] A.B. Cortesi, B.L. Smith, B. Sigg, S. Banerjee, "Numerical Investigation of the Scalar Probability Density Function Distribution in Neutral and Stably Stratified Mixing Layers," International Journal of Physics Fluids, Vol.13, Issuue.4, pp.927-950, 2001.

[11] S.B Doma, N.A El-Nohy, M.A Salem, "Ground States of Few Electron Atoms in a Strong Magnetic Field Using Diffusion Monte Carlo Method," International Journal of Scientific Research in Physics and Applied Sciences, Vol.8, Issue.1, pp. 17, 2020.

\section{AUTHORS PROFILE}

Daniel Katz Bonello, MSc. Graduate in Control and Automation Engineering at Universidade Paulista - UNIP (2016), Campinas-Brazil, also holds a MSc degree in Electrical Enginerring (2020) at UNICAMP. Research Interests: Computer Vision, Image Processing, Machine Leraning, Automation Strategies.

Yuzo Iano, PhD. Prof. Yuzo Iano is the head and founder of the Laboratory of Visual Communications since 1972. He obtained his BSc (1972), MSc (1974) and $\mathrm{PhD}$ (1986) in Electrical Engineering at UNICAMP, SP-Brazil. Research Interests: Digital Signal Processing, Pattern Recognition, Internet of Things, Digital TV.

Umberto Bonello Neto, BSc. Graduate in Building Technology at UNICAMP, SP-Brazil (1987), is MSc candidate at UNICAMP. Research Interests: Process Control Strategies, Fieldbus Technology, Deep Learning, PCB design.

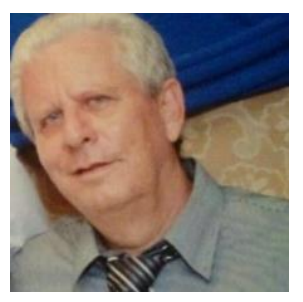

